\title{
A critical assessment of the medication-related osteonecrosis of the jaw classification in stage I patients: a retrospective analysis
}

\author{
Oliver Ristow, Lena Hürtgen, Julius Moratin, Maximilian Smielowski, Christian Freudlsperger, \\ Michael Engel, Jürgen Hoffmann, Thomas Rückschloß \\ Department of Oral and Maxillofacial Surgery, University of Heidelberg, Heidelberg, Germany
}

\begin{abstract}
J Korean Assoc Oral Maxillofac Surg 2021;47:99-111)
Objectives: It is unclear whether the extent of intraoral mucosa defects in patients with medication-related osteonecrosis of the jaw indicates disease severity. Therefore, this study investigated whether mucosal lesions correlate with the true extent of osseous defects in stage I patients.

Materials and Methods: Retrospectively, all patients with stage I medication-related osteonecrosis of the jaw who underwent surgical treatment between April 2018 and April 2019 were enrolled. Preoperatively, the extent of their mucosal lesions was measured in clinical evaluations, and patients were assigned to either the visible or the probeable bone group. Intraoperatively, the extent of necrosis was measured manually and with fluorescence. Results: Fifty-five patients (36 female, 19 male) with 86 lesions (46 visible bone, 40 probeable bone) were enrolled. Intraoperatively, the necrotic lesions were significantly larger $(P<0.001)$ than the preoperative mucosal lesions in both groups. A significant $(P<0.05)$ but very weak $\left(R^{2}<0.2\right)$ relationship was noted between the extent of the mucosal lesions and the necrotic bone area.

Conclusion: Preoperative mucosal defects (visible or probeable) in patients with medication-related osteonecrosis of the jaw do not indicate the extent of bone necrosis or disease severity.
\end{abstract}

Key words: Medication-related osteonecrosis of the jaw, Bisphosphonate-related osteonecrosis of the jaw, Antiresorptiva-related osteonecrosis of the jaw, Definition, Classification

[paper submitted 2020. 11. 15 / revised 2020. 12. 16 / accepted 2020. 12. 17]

\section{Introduction}

Since its initial description ${ }^{1}$, the incidence of medicationrelated osteonecrosis of the jaw (MRONJ) and the number of related publications have increased rapidly. As this serious disease ${ }^{2}$ has been studied by clinicians and scientists, controversies have arisen between medical societies ${ }^{3-6}$.

The Advisory Task Force on Bisphosphonate-Related Osteonecrosis of the Jaw from the American Association of Oral and Maxillofacial Surgeons (AAOMS) has made great efforts to present an accepted and widely used definition

\section{Thomas Rückschloß \\ Department of Oral and Maxillofacial Surgery, University of Heidelberg, Im Neuenheimer Feld 400, D-69120 Heidelberg, Germany \\ TEL: +49-6221-56-37314 \\ E-mail: thomas.rueckschloss@med.uni-heidelberg.de \\ ORCID: https://orcid.org/0000-0002-7955-9194}

(C) This is an open-access article distributed under the terms of the Creative Commons Attribution Non-Commercial License (http://creativecommons.org/ licenses/by-nc/4.0/), which permits unrestricted non-commercial use, distribution, and reproduction in any medium, provided the original work is properly cited. Copyright (C) 2021 The Korean Association of Oral and Maxillofacial Surgeons. All rights reserved. of $\mathrm{MRONJ}^{7-9}$. The following definition is the current gold standard for MRONJ worldwide: the presence of exposed jawbone or bone that can be probed for at least 8 weeks in a patient receiving antiresorptive therapy who has not received radiotherapy to the head or neck. Furthermore, AAOMS developed a four-stage disease classification scale (stage 0 to III) that is regarded as the standard for deriving treatment recommendations and creating comparability in the nomenclature of epidemiological data 9 .

However, the AAOMS staging system has also been the subject of controversy. The absolute goal of a staging system should be to depict the extent of the disease, derive suitable therapeutic options, and make prognostic assessments. However, the AAOMS staging system, which is driven by clinical inspectorial examinations, might fail to detect the actual extent of bone involvement in MRONJ, raising the risk of assigning patients to inappropriate treatments ${ }^{10,11}$. In particular, AAOMS stage $\mathrm{I}^{9}$ is misleading. Because an early stage implies a good prognosis, the AAOMS recommends that stage I patients be treated with a non-surgical, conserva- 
tive approach. However, the size of the mucosal defect (sinus tract or bones exposed over a large area) does not allow conclusions to be drawn about the true extent of the necrosis or the appropriate therapy recommendation or prognosis. Indeed, few data are available to support MRONJ staging or the concerns about it. Therefore, it is not surprising that many working groups have attempted to investigate the relationship between the staging and the true extent of necrosis by using radiological diagnostics to detect early signs of MRONJ or assess the real extent of the disease ${ }^{12-16}$. Unfortunately, there is no consensus on the efficacy of different imaging modalities in assessing the real extent of necrotic bone area ${ }^{17,18}$. Furthermore, inconsistent results have been found, including both over- and underestimation of the extent of MRONJ when comparing the AAOMS stages with various imaging modalities $^{19}$. At present, it seems that determining the true extent of necrosis is only possible intraoperatively using the judgment of experienced surgeons or fluorescence-based methods ${ }^{20-22}$.

To date, no clinical data investigating the connection between the size of a mucosal defect and the extent of necrosis have been published. Before it makes sense to question a clinically established classification to improve it, it is urgently necessary to provide evidence that the classification is clinically relevant. Therefore, our aims in this study were to determine whether the extent of a mucosal lesion correlates with the bony lesion in stage I patients. We also wanted to assess possible intragroup differences in the clinical manifestation of necrosis (probeable vs visible bone). We hypothesize that the extent of the mucosal lesion bears no relationship to the bony lesion and that whether the necrotic bone is visible or probeable is clinically irrelevant.

\section{Materials and Methods}

\section{Patients}

This study was approved by the Ethics Committee of the University of Heidelberg (No. S-401/2020) and carried out according to the current version of the Declaration of Helsinki. We designed and implemented a retrospective, singlecenter, cohort study and consecutively enrolled a sample derived from the population of subjects who received a working diagnosis of MRONJ and fulfilled set inclusion criteria at the Department of Maxillofacial Surgery, University of Heidelberg, between April 2018 and April 2019. The criteria were as follows: (1) patients with MRONJ stage I (exposed, necrotic bone or sinus tract with probes to bone; no symp- toms or evidence of infection at the time of first presentation in the anamnesis or in the further treatment course) according to the AAOMS criteria ${ }^{9}$; (2) ongoing or previous history of antiresorptive treatment; (3) no preceding surgical treatment for MRONJ; and (4) the presence of data regarding the extent of the preoperative mucosal lesions and intraoperative osseous necrotic lesions. The exclusion criteria were as follows: (1) history of head or neck radiation and (2) metastatic bone disease of the maxillofacial region.

\section{Intervention/data collection}

All patients with a history of antiresorptive intake routinely take part in a weekly consulting hour at our unit. Therefore, follow-up examinations and radiographic documentation were performed according to our internal unit's standardized protocols, and they were always conducted by the same investigators (O.R., T.R., and M.S.).

\section{1) Preoperative data collection (T0)}

On the day of surgery planning in our interdisciplinary consulting hours, approximately four weeks prior to surgery (T0), a standardized clinical examination was performed. In addition, a cone-beam computed tomography (CBCT) image and a standardized measurement of the extent of the mucosal MRONJ lesion were acquired from all patients with visibly exposed bone. The measurements were performed manually in the anteroposterior (parallel to the sagittal and occlusal plane) and transversal directions (parallel to the coronal and occlusal plane) using digital calipers. For reasons of practicality, patients with a sinus tract that probed to the bone did not receive a measurement of the size of the opening. In those patients, the extent of sinus tract was defined as $0 \mathrm{~mm}$ in the anteroposterior and transversal directions. A GALILEOS Comfort was used as the CBCT scanner (98 kV at 3-8 mA pulsed operation, spherical volume of $15.4 \mathrm{~cm}$, scanning time of $14 \mathrm{~s}$, isotopic voxel size of $0.25 \mathrm{~mm}$; Dentsply Sirona, Bensheim, Germany). Each image dataset was reconstructed in the sagittal and coronal plane using the software provided by the manufacturer. Data acquisition followed the standardized, routine protocol used in our unit; during the scan, patients adopted a standing position with the Frankfurt horizontal plane parallel to the floor.

2) Surgical intervention (T1)

All surgical procedures were performed under general anesthesia by one surgeon (O.R.) following a previously 
published surgical protocol ${ }^{22,23}$ and adhering to a standardized, intra-institutional protocol from the German guidelines for $\mathrm{MRONJ}^{5}$ : (1) adjunctive antibiotic therapy; (2) complete removal of necrotic bone; (3) smoothing of sharp bone edges (modeling osteotomy); and (4) secure and well vascularized plastic wound closure.

Specifically, all patients were preoperatively treated with oral antibiotics (amoxicillin/clavulanic acid 875/125 mg 1-01; Clindamycin $600 \mathrm{mg} 1-1-1$ in case of penicillin allergy) for five days to prevent the development of infection at the time of surgery. A "drug holiday" was performed for four weeks before and after surgery in cases in which it was justifiable according to oncological or osteological considerations. After elective inpatient admission one day prior to surgery, intravenous antibiotic treatment began (sulbactam/ampicillin 500/1,000 mg 1-1-1; Clindamycin $600 \mathrm{mg} \mathrm{1-1-1} \mathrm{in} \mathrm{case} \mathrm{of}$ penicillin allergy). On the day of surgical intervention, after anesthetic induction, a second manual measurement of the extent of the mucosal lesion was performed using a procedure similar to T0 (Fig. 1. A, 1. B) with digital calipers. The following procedure was performed during the operation: after surgical bone exposure, the VELscope system Vx (LED Dental, White Rock, BC, Canada) was used to induce and visualize auto-fluorescence of the jawbone. As described previously by our group ${ }^{21,22}$, this method allows the expansion of necrotic bone to be represented with great clinical accuracy. Under the light of the VELscope, viable bone shows a greenish auto-fluorescence, whereas necrotic bone areas show no or only very pale autofluorescence. After that, the transition zones from the necrotic bone area to the healthy jawbone were marked with a surgical skin marker.(Fig. 1. C, 1. D)

That was followed by another clinical measurement of the extent of the necrotic area with the digital calipers. Next, the bone resection was performed until the bright fluorescence of healthy bone was observed. Additionally, resection was performed with respect to the macroscopic bone findings (color,

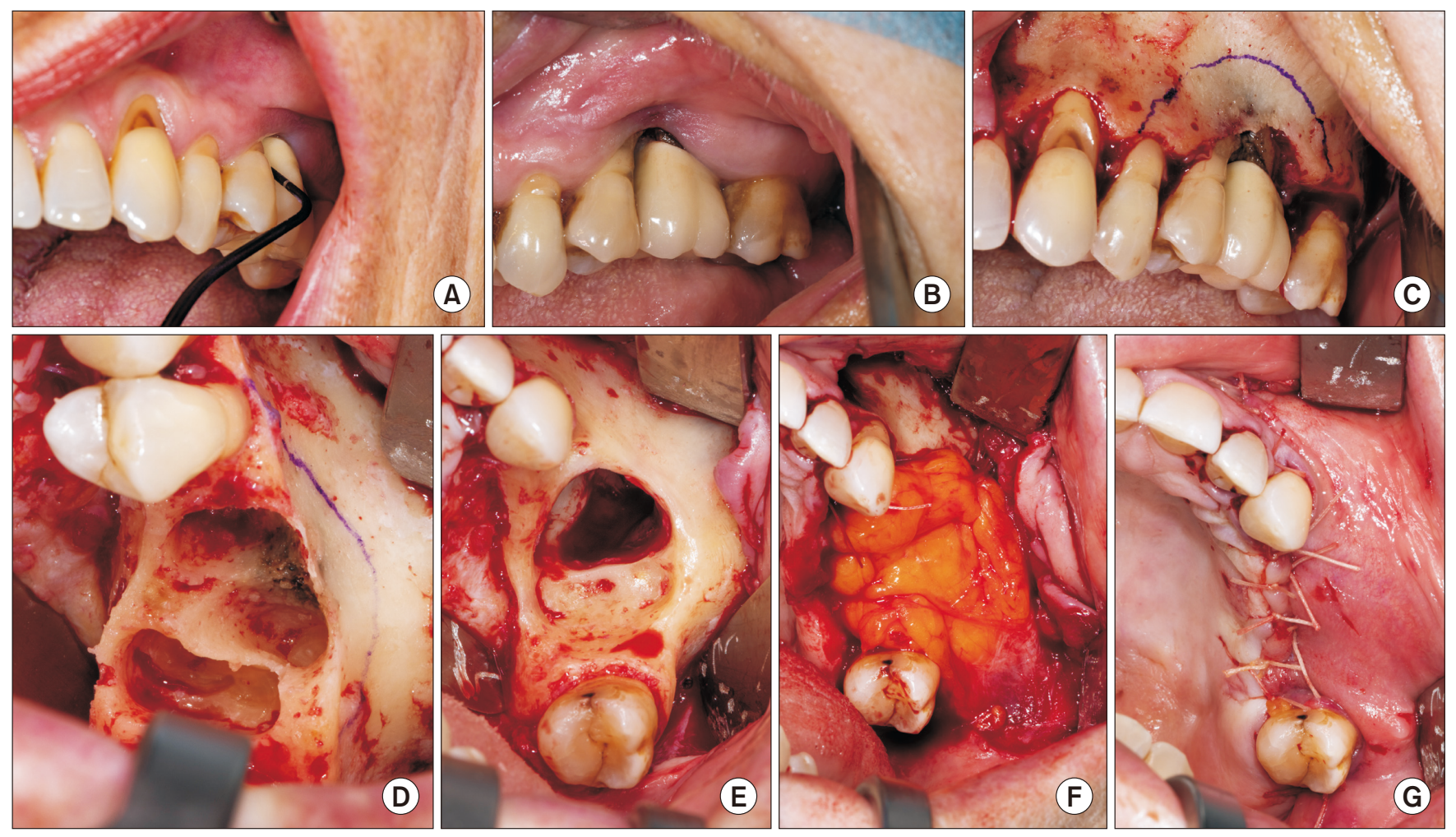

Fig. 1. A 64-year-old female patient; underlying disease: osseous metastasized breast carcinoma; antiresorptive therapy: zoledronate $4 \mathrm{mg}$ every 4 weeks intravenously over 4 years; presentation with unspecific symptoms of the upper jaw (recurrent pain and signs of inflammation) and increased probing depths over 12 months. A, B. Preoperative intraoral findings with probeable bone at first sight (A) and visible bone after carefully pushing back the mucosa (B). C. Intraoperative findings of the necrotic area after subperiosteal preparation: macroscopic marking of the necrosis using a pen after florescence visualization. D. After extracting necrosis-adjacent teeth (\#25 and \#26), a cone-like necrotic extension in the alveolus up to the maxillary sinus became visible. E. Intraoperative findings after resection of the necrotic bone and smoothing of all bone edges: the maxillary sinus is opened. F. Tension-free wound closure using double-layer closure techniques with a pedicled buccal fat flap. G. The muco-periosteal layer.

Oliver Ristow et al: A critical assessment of the medication-related osteonecrosis of the jaw classification in stage I patients: a retrospective analysis. J Korean Assoc Oral Maxillofac Surg 2021 
texture, and bone hardness). Subsequently, sharp bone edges were smoothed using milling cutters and diamond drills. (Fig. 1. E) A tension-free wound closure was achieved using double-layer closure techniques with a mylohyoid flap in the lower jaw and pedicled buccal fat flap in the upper jaw ${ }^{23}$.(Fig. 1. F, 1. G) After surgery, intravenous antibiotic treatment was continued until inpatient discharge on the fifth postoperative day. After discharge, oral antibiotic treatment was continued for a further seven days. Standardized suture removal occurred 14 days after surgery.

\section{3) Postoperative follow-up 8 weeks after surgery (T2)}

Eight weeks after the operation, the patients returned to our special consultation for regular follow-up. At that appointment, a second CBCT was performed using procedures similar to those at T0.

\section{Data analysis of CBCT}

Data analysis was performed by two investigators (T.R. and L.H.) using the IPlan Cranial software tool (Brainlab, Feldkirchen, Germany), which is commonly used for treatment planning in cranio-, oral- and maxillofacial surgery. To standardize the measurement of the data sets, the preoperative Dicom dataset (acquired from T0 CBCT) was aligned to a three-dimensional Cartesian coordinate system and oriented to the occlusal and sagittal planes.

As a next step, the Image Fusion Tool in IPlan Cranial was used to voxel-wisely register the postoperative Dicom dataset (acquired from the $\mathrm{T} 2 \mathrm{CBCT}$ ) to the aligned preoperative da- taset on the basis of the part of the jaw that was not affected by the surgical intervention.(Fig. 2)

We measured the resection defect using those preoperative and postoperative CBCT images by subtracting the postoperative DICOM dataset from the preoperative one, which produced an exact visualization and measurement of the resection defect. For each measurement, the anteroposterior and transversal extent of the necrotic lesion was determined parallel to the same planes used in the clinical measurements.

We also examined whether the resection defect extended beyond the region of alveolar bone, which would postoperatively make it a stage III MRONJ lesion.

\section{Summary of measured parameters}

The following measurements (each in the anteroposterior and transverse directions) were obtained in this study: (1) extent of the mucosal MRONJ lesion 4 weeks prior to surgery, as measured by digital calipers; (2) extent of the mucosal MRONJ lesion on the day of surgery using digital calipers; (3) extent of the osseous necrotic MRONJ lesion, as measured intraoperatively using auto-fluorescence; and (4) extent of resection defect, measured by analyzing preoperative and postoperative CBCT scans.

\section{Statistical analysis}

All statistical analyses were performed using IBM SPSS Statistics software (ver. 27; IBM, Armonk, NY, USA). In addition to the descriptive evaluation (arithmetic mean, stan-
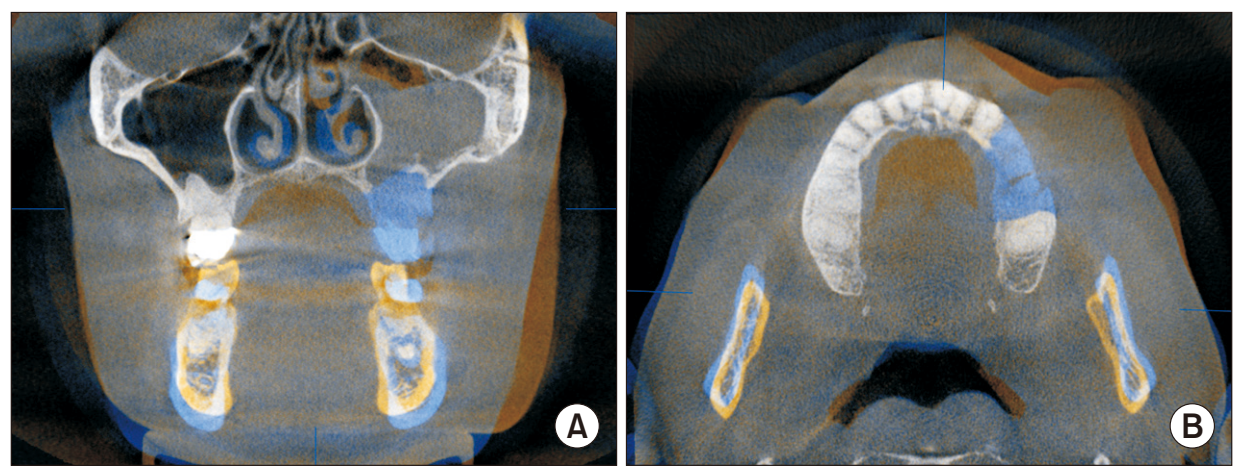

Fig. 2. Fused preoperative (blue) and postoperative (orange) cone-beam computed tomography scans in coronal (A) and axial (B) views: the resection defect in the upper jaw on the left side is highlighted in blue. Note: the swelling of the mucosa in the maxillary sinus. However, no bone reaction (such as bone loss or resorption, osteolysis, or osteosclerosis) could be found. Therefore, this patient was preoperatively classified as stage I. However, postoperatively the lesion should certainly have been classified as stage III because the floor of the maxillary sinus had to be removed. Preoperative clinical information (size of the mucosal defect) offers no conclusion about the severity of the bone disease.

Oliver Ristow et al: A critical assessment of the medication-related osteonecrosis of the jaw classification in stage I patients: a retrospective analysis. J Korean Assoc Oral Maxillofac Surg 2021 
dard deviation), we performed two-tailed $t$-testing of paired samples from each time of measurement. A subgroup analysis was performed by dividing the patients into two groups (visible bone group and probeable bone group) defined according to current AAOMS classification data ${ }^{9}$. The visible bone group contained patients with exposed, necrotic bone visible to the naked eye on the day of inclusion. The probeable bone group contained patients without visible necrotic bone but with necrotic bone that could be probed via a sinus tract using a standardized World Health Organization probe. For this purpose, a two-sample $t$-test was performed. A linear regression model was calculated to quantify whether the amount of intraorally exposed bone, the intraoperative extent of necrotic bone, and the size of resection defect correlated with one another. The examination of the residuals for normality was carried out on the basis of histograms and pivot point-chartscharts. $P$-values less than 0.05 were regarded as statistically significant.

\section{Results}

\section{Patient data}

In total, 55 Caucasian patients with 86 MRONJ lesions were enrolled in this study (36 females [mean age, 68.2 \pm 8.5 years] and 19 males [mean age, $72.5 \pm 10.4$ years]).

Table 1. Descriptive statistics indicating the extent of the lesions

\begin{tabular}{|c|c|c|c|c|}
\hline Group & Direction & Measurement & No. of patients & Value (mm) \\
\hline \multirow[t]{8}{*}{ Visible bone group } & \multirow[t]{4}{*}{ Anteroposterior } & Mucosal lesion 4 weeks prior to surgery & 46 & $11.2 \pm 9.0(1.0-36.0)$ \\
\hline & & Mucosal lesion on day of surgery & 46 & $10.2 \pm 8.3(1.0-30.0)$ \\
\hline & & Osseous necrotic lesion intraoperatively & 46 & $27.4 \pm 12.2(10.0-75.0)$ \\
\hline & & Resection defect (measured radiologically) & 31 & $33.6 \pm 8.6(17.6-53.5)$ \\
\hline & \multirow[t]{4}{*}{ Transversal } & Mucosal lesion 4 weeks prior to surgery & 46 & $7.7 \pm 6.5(1.0-24.0)$ \\
\hline & & Mucosal lesion on day of surgery & 46 & $7.1 \pm 5.7(1.0-20.0)$ \\
\hline & & Osseous necrotic lesion intraoperatively & 46 & $17.1 \pm 7.2(8.5-50.0)$ \\
\hline & & Resection defect (measured radiologically) & 31 & $21.8 \pm 6.1(11.3-32.8)$ \\
\hline \multirow[t]{8}{*}{ Probeable bone group } & \multirow[t]{4}{*}{ Anteroposterior } & Mucosal lesion 4 weeks prior to surgery & 40 & 0 \\
\hline & & Mucosal lesion on day of surgery & 40 & 0 \\
\hline & & Osseous necrotic lesion intraoperatively & 40 & $20.8 \pm 9.4(4.0-37.0)$ \\
\hline & & Resection defect (measured radiologically) & 32 & $29.0 \pm 10.5(7.8-45.8)$ \\
\hline & \multirow[t]{4}{*}{ Transversal } & Mucosal lesion 4 weeks prior to surgery & 40 & 0 \\
\hline & & Mucosal lesion on day of surgery & 40 & 0 \\
\hline & & Osseous necrotic lesion intraoperatively & 40 & $15.4 \pm 9.0(3.0-55.0)$ \\
\hline & & Resection defect (measured radiologically) & 32 & $18.0 \pm 6.5(4.0-30.1)$ \\
\hline
\end{tabular}

Values are presented as mean \pm standard deviation (range).

Oliver Ristow et al: A critical assessment of the medication-related osteonecrosis of the jaw classification in stage I patients: a retrospective analysis. J Korean Assoc Oral Maxillofac Surg 2021

Table 2. Two-tailed $t$-test between different time points

\begin{tabular}{|c|c|c|c|c|c|c|c|}
\hline Group & Direction & Measurement (1) & Measurement (2) & Value (mm) & $t$ & $\mathrm{df}$ & $P$ (2-tailed) \\
\hline \multirow[t]{6}{*}{$\begin{array}{l}\text { Visible } \\
\text { bone group }\end{array}$} & \multirow[t]{3}{*}{ Anteroposterior } & $\begin{array}{l}\text { Mucosal lesion } 4 \text { weeks } \\
\text { prior to surgery }\end{array}$ & $\begin{array}{l}\text { Mucosal lesion on day } \\
\text { of surgery }\end{array}$ & $0.98 \pm 4.23$ & 1.57 & 45 & 0.124 \\
\hline & & $\begin{array}{l}\text { Mucosal lesion on day } \\
\text { of surgery }\end{array}$ & $\begin{array}{l}\text { Osseous necrotic lesion } \\
\text { intraoperatively }\end{array}$ & $-17.24 \pm 11.70$ & -9.99 & 45 & $0.000^{*}$ \\
\hline & & $\begin{array}{l}\text { Osseous necrotic lesion } \\
\text { intraoperatively }\end{array}$ & $\begin{array}{l}\text { Resection defect } \\
\text { (measured radiologically) }\end{array}$ & $-7.52 \pm 4.09$ & -10.23 & 30 & $0.000 *$ \\
\hline & \multirow[t]{3}{*}{ Transversal } & $\begin{array}{l}\text { Mucosal lesion } 4 \text { weeks } \\
\text { prior to surgery }\end{array}$ & $\begin{array}{l}\text { Mucosal lesion on day } \\
\text { of surgery }\end{array}$ & $0.65 \pm 3.40$ & 1.30 & 45 & 0.200 \\
\hline & & $\begin{array}{l}\text { Mucosal lesion on day } \\
\text { of surgery }\end{array}$ & $\begin{array}{l}\text { Osseous necrotic lesion } \\
\text { intraoperatively }\end{array}$ & $-9.98 \pm 7.28$ & -9.30 & 45 & $0.000^{*}$ \\
\hline & & $\begin{array}{l}\text { Osseous necrotic lesion } \\
\text { intraoperatively }\end{array}$ & $\begin{array}{l}\text { Resection defect } \\
\text { (measured radiologically) }\end{array}$ & $-6.05 \pm 3.45$ & -9.75 & 30 & $0.000^{*}$ \\
\hline \multirow[t]{2}{*}{$\begin{array}{l}\text { Probeable } \\
\text { bone group }\end{array}$} & Anteroposterior & $\begin{array}{l}\text { Osseous necrotic lesion } \\
\text { intraoperatively }\end{array}$ & $\begin{array}{l}\text { Resection defect } \\
\text { (measured radiologically) }\end{array}$ & $-7.86 \pm 5.02$ & -8.86 & 31 & $0.000^{*}$ \\
\hline & Transversal & $\begin{array}{l}\text { Osseous necrotic lesion } \\
\text { intraoperatively }\end{array}$ & $\begin{array}{l}\text { Resection defect } \\
\text { (measured radiologically) }\end{array}$ & $-4.26 \pm 2.03$ & -11.90 & 31 & $0.000^{*}$ \\
\hline
\end{tabular}

Values are presented as mean \pm standard deviation.

${ }^{*} P<0.05$ (two-tailed $t$-test between different timepoints/parameters of measurement).

Oliver Ristow et al: A critical assessment of the medication-related osteonecrosis of the jaw classification in stage I patients: a retrospective analysis. J Korean Assoc Oral Maxillofac Surg 2021 
Nine patients who were included suffered from osteoporosis, and 46 had an underlying malignancy with metastasis to or primary focus (such as multiple myeloma) on the bone (prostate, 12; breast, 20; multiple myeloma, 5; kidney cell carcinoma, 4; and others, 5).

Of the 55 patients included, 19 were treated with zoledronate, 2 with ibandronat, and 4 with alendronate. Twentyfive patients were treated with denosumab. The remaining 5 patients reported subsequent or alternating intake of bisphosphonates and denosumab. The mean duration of intake for the antiresorptive drugs prior to treatment was $49.3 \pm 41.5$ months (range, 8-224 months).

Thirty-one patients showed only one MRONJ lesion, 23 patients had two, and 3 patients had three. Thirty-five lesions were located in the upper jaw, and 51 lesions were located in the lower jaw.

Of the 86 stage I MRONJ ${ }^{9}$ lesions, 46 patients had bone visibly exposed to the oral cavity (visible bone group), and 40 lesions could be probed to bone through the sinus tract (probeable bone group).

\section{Extent of mucosal lesions, intraoperative necrotic lesions}

Detailed results are given in Tables 1 and 2 and Fig. 3. In the visible bone group, the mucosal lesion in both the anteroposterior and transversal directions decreased from the day of inclusion (T0) (anteroposterior dimension, 11.2 $\pm 9.0 \mathrm{~mm}$; transversal, $7.7 \pm 6.5 \mathrm{~mm}$ ) to the day of surgery (anteroposterior dimension, $10.2 \pm 8.3 \mathrm{~mm}$; transversal, $7.1 \pm 5.7 \mathrm{~mm}$ ). However, those differences are not significant.

In the probeable bone group, mucosal healing did not occur in any patient from $\mathrm{T} 0$ until surgery.

When we compared the preoperative mucosal lesion with the extent of the necrotic osseous lesion in the visible bone group, the extent of the osseous lesion (anteroposterior dimension, $27.4 \pm 12.2 \mathrm{~mm}$; transversal, $17.1 \pm 7.2 \mathrm{~mm}$ ) was significantly $(P<0.001)$ larger than the mucosal lesion (anteroposterior dimension, $10.2 \pm 8.3 \mathrm{~mm}$; transversal, $7.1 \pm 5.7 \mathrm{~mm}$ ).

In the probeable bone group, extensive necrotic lesions also appeared intraoperatively (anteroposterior dimension, $20.8 \pm 9.4 \mathrm{~mm}$; transversal, $15.4 \pm 9.0 \mathrm{~mm}$ ).

\section{Resection defects}

Detailed results are presented in Tables 1 and 2 and Fig. 3. In the visible bone group, the resection defect (anteroposterior dimension, 33.6 $\pm 8.6 \mathrm{~mm}$; transversal, $21.8 \pm 6.1 \mathrm{~mm}$ ) was significantly larger (anteroposterior dimension, $\mathrm{T}(30)=-10.23$, $P<0.001$; transversal, $\mathrm{T}(30)=-9.75, P<0.001)$ than the necrotic area (anteroposterior dimension, $27.4 \pm 12.2 \mathrm{~mm}$; transversal, 17.1 $\pm 7.2 \mathrm{~mm})$.

In the probeable bone group, the resection defect (anteroposterior dimension, $29.0 \pm 10.5 \mathrm{~mm}$; transversal, 18.0 \pm 6.5 $\mathrm{mm}$ ) was also significantly larger (anteroposterior dimension, $\mathrm{T}(31)=8.86, P<0.001$; transversal, $\mathrm{T}(31)=-11.90, P<0.001)$
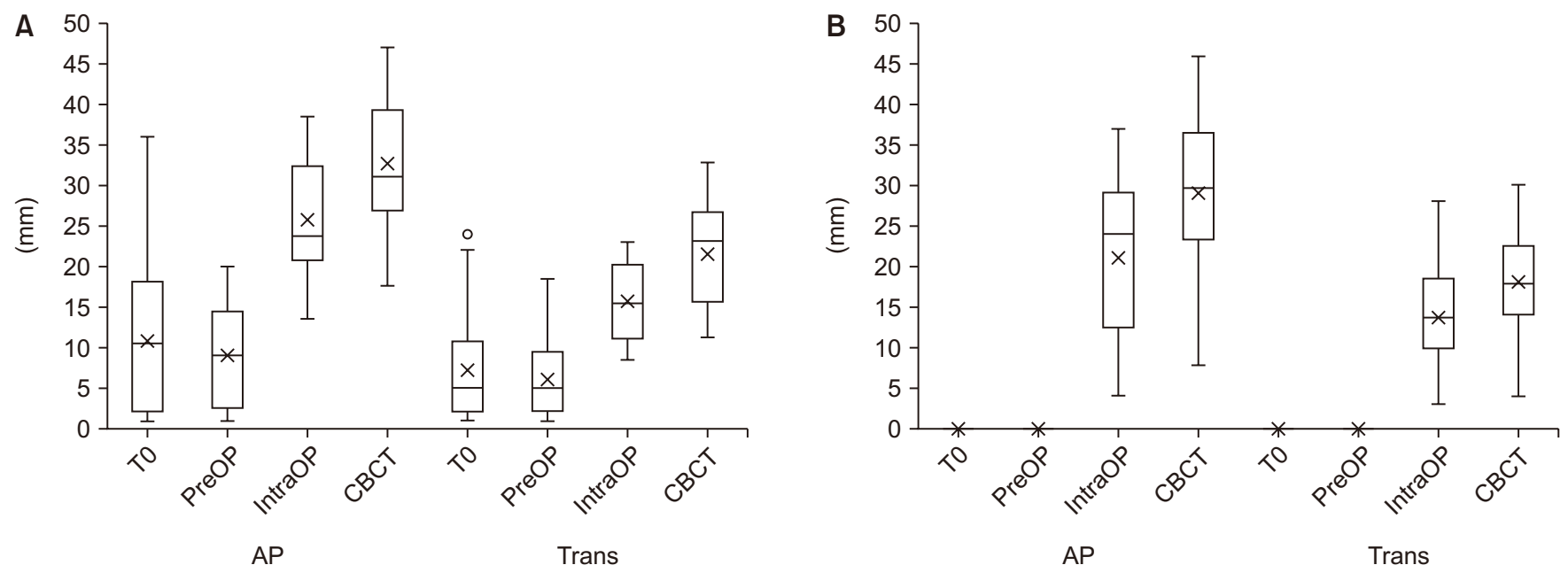

Fig. 3. Boxplot comparing the anteroposterior (AP) and transversal (Trans) extent of: the mucosal lesion at presentation (T0), the mucosal lesion on the day of surgery (PreOP), the extent of the necrotic bone intraoperatively (IntraOP), and the extent of the resection defect measured by cone-beam computed tomography (CBCT) in the visible bone group (A) and probeable bone group (B).

Oliver Ristow et al: A critical assessment of the medication-related osteonecrosis of the jaw classification in stage I patients: a retrospective analysis. J Korean Assoc Oral Maxillofac Surg 2021 
than the necrotic osseous area (anteroposterior dimension, $20.8 \pm 9.4 \mathrm{~mm}$; transversal, $15.4 \pm 9.0 \mathrm{~mm}$ ).

The CBCT analysis showed that in 6 lesions in 3 patients, the resection defects exceeded the region of alveolar bone and could thus postoperatively be considered stage III lesions.

\section{Comparison between groups}

Detailed results are given in Table 3. When we compared the two groups using a two-sample $t$-test, the extent of the osseous necrotic lesion in the anteroposterior direction turned out to be significantly $(\mathrm{T}(84)=2.78 ; P=0.007)$ larger in the visible bone group. No significant difference between the two groups was found for the extent of the osseous lesion in the transversal direction $(\mathrm{T}(84)=0.96 ; P=0.342)$.

\section{Relationships}

Table 4 depicts the results of the linear regression analysis of the measurements in both groups.

Using the extent of the mucosal lesion on the day of surgery as the predictor and the extent of the osseous necrotic lesion measured intraoperatively as the dependent variable in the anteroposterior direction in the visible bone group, the overall regression model was significant $(\mathrm{T}(45)=2.872$, $P=0.006)$. Thus, the extent of the mucosal lesion predicted the extent of necrotic bone $(\mathrm{B}=0.583)$. However, that linear regression model explains only $15.8 \%$ of the variance in the data $\left(R^{2}=0.158\right)$. The finding for the extent of necrotic bone and the resection defect was the same.

\section{Discussion}

Even though great progress has been made in diagnosing and treating MRONJ in recent years, diagnosis (especially early cases) and staging remain challenging, partly because the diagnostic procedure is not standardized, and the AAOMS classification does not cover all manifestations of MRONJ $^{11,19}$.

Compared with the 2009 version $^{7,8}$, the most recent version of the AAOMS consensus paper has widened the definition of $\mathrm{MRONJ}^{9}$. This amendment was triggered by several studies that found that patients can suffer from MRONJ without visually detectable necrotic bone (non-exposed MRONJ, including intraoral fistula, mandibular fracture, dentally unexplained pain, and swelling) and would therefore not fulfill the

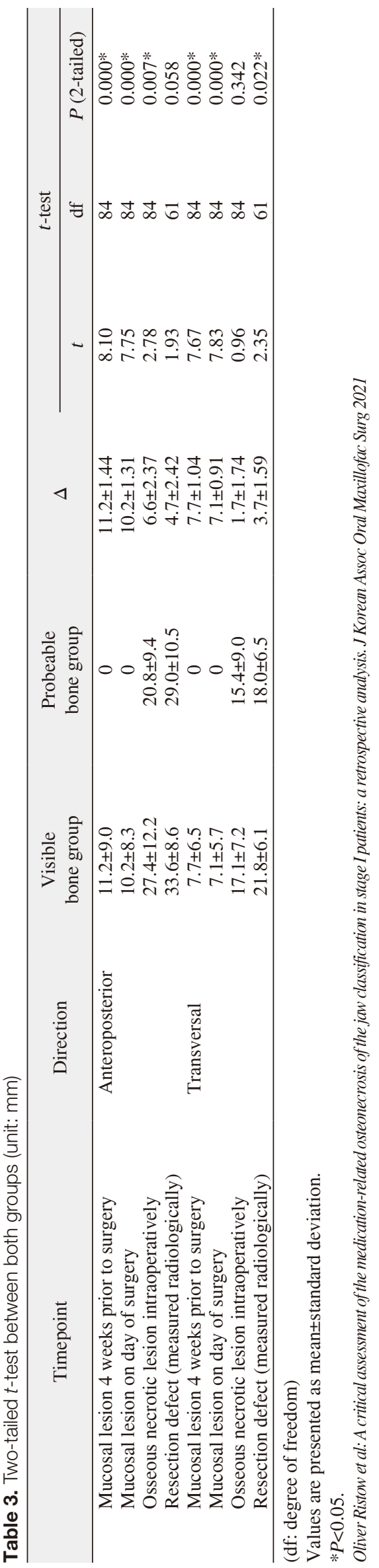




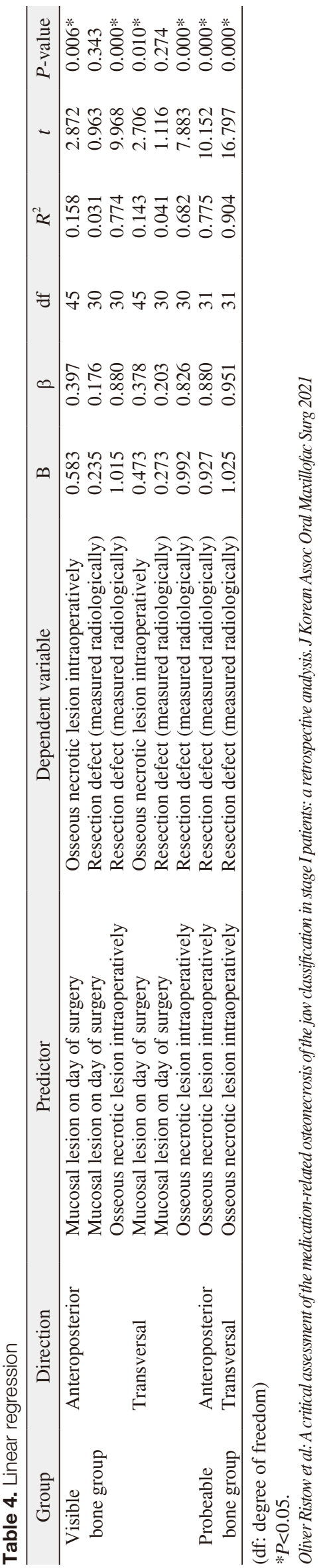

initial definition of MRONJ ${ }^{10,12,13,24,25}$. Therefore, the modified definition in the latest AAOMS consensus paper includes patients who present with bone probeable through a sinus tract ${ }^{9}$. This change is an important step forward because different interpretations of "bone exposure" had led some authors to diagnose probeable bone as MRONJ, whereas others did not. That disagreement might partly explain the different prevalence figures in recent epidemiological studies ${ }^{19}$.

However, the current staging system still suffers from several inconsistencies. In its 2009 consensus paper ${ }^{8}$, AAOMS added a "stage 0 " to its classification system for subjects with the non-exposed variant of MRONJ (no clinical evidence of necrotic bone but nonspecific clinical findings, radiographic changes, and symptoms). This stage is accompanied by nonspecific symptoms (odontalgia, bone pain in the jaw, loosening of teeth, and others). Unfortunately, the core definition of MRONJ remains unchanged, and AAOMS continues to focus on the clinical evidence of long-standing bony exposure $^{11}$. Consequently, patients with non-exposed MRONJ without probeable bone do not have MRONJ as it is currently defined. This paradox has been highlighted by many authors calling for urgent change ${ }^{13,26-28}$.

In a hierarchical staging system such as the AAOMS classification (stage 0 -III), both clinicians and patients should be able to assume that the stages differ in terms of the extent or severity of disease they represent. However, AAOMS has made no statement about whether their stages correlate with differences in the extent of necrotic bone area ${ }^{9}$. That failure was precisely the starting point for this study.

In this study, we have shown that the intraoperatively detected areas of necrotic bone were significantly larger than the preoperative visible mucosal lesions in both the visible and probeable bone groups. However, we also showed that the intraoperatively detected necrotic osseous lesions in stage I patients ${ }^{9}$ with mucosal defects and visible bone were significantly larger than those in patients with preoperative probeable bone. Furthermore, our linear regression model shows a weak but significant correlation between the extent of the mucosal lesion and the necrotic bone area in the anteroposterior direction in patients who presented with visible bone preoperatively.

However, the very low correlation coefficients $\left(R^{2}<0.2\right)$ indicate that our regression models cannot be used to predict the exact extent of a necrotic bony lesion from the extent of a mucosal lesion. The scattering around the regression line is far too large for that. Instead, it must be assumed that other factors influence the extent of both the necrotic and mucosal 
lesions. Furthermore, the direction of influence is unclear. For future studies, we highly recommend the use of multivariate regression analyses in a larger population.

A further linear regression model between the preoperative extent of the mucosal lesion and the resection defect did not show significant results. In other words, preoperative patient presentation with visible or probeable bone does not predict the extent of the underlying necrotic osseous lesion. However, large areas of exposed bone might indicate that necrosis is extensive, perhaps because a small mucosal lesion protects necrotic bone from prolonged exposure to bacteria, which limits secondary infections and disease progression.

Furthermore, we have shown that the resection defects were significantly larger than the mucosal lesions, although no regression was detected between the extent of the mucosal lesion and the surgically induced defect. That the resection defect is obviously larger than the mucosal and osseous lesions is not surprising; it is a result of the necessary extraction of adjacent teeth and the smoothing of sharp bone edges after the removal of the necrotic bone. However, our results suggest that it is impossible to use mucosal defects and AAOMS stages $^{9}$ to predict the effort needed for the surgical management of MRONJ.

Furthermore, for 6 lesions classified as stage I preoperatively, resections beyond the alveolar bone were needed, meaning that those lesions were postoperatively classified as stage III.

To the best of our knowledge, no previous study has investigated the questions addressed herein. A partly comparable study was conducted by Assaf et al. ${ }^{29}$, who compared the extent of MRONJ, as detected by Tc-99m-methylene diphosphonate bone scintigraphy, with the intraoperative extent of the disease. They found that the true extent of osseous lesions, as determined by surgery, was significantly underestimated by clinical examination but not by bone scintigraphy ${ }^{29}$. Their results underline the importance of imaging in diagnosing and managing MRONJ.

A criticism from surgeons who treat according to the AAOMS treatment recommendations for MRONJ will certainly be that the patients in the present study who presented with MRONJ stage $\mathrm{I}^{9}$ were surgically treated. There is currently great controversy between international professional societies regarding the AAOMS treatment recommendations ${ }^{9}$. For stages I and II, AAOMS recommends non-surgical treatment ${ }^{9}$ such as symptomatic treatment with oral antibiotics, oral antibacterial mouth rinse, pain control, and regular clinical follow-up. Surgical debridement or resection of necrotic bone is recommended for stage II MRONJ patients'. Similar recommendations were also made by Khan et al. ${ }^{30}$ on behalf of the International Osteonecrosis of the Jaw Task Force. They based their treatment recommendations on the disease stage and size of the lesion. In their view, conservative therapy should be continued until obvious progression of the disease occurs, pain cannot be controlled by conservative means, or antiresorptive therapy is discontinued by the treating oncologist ${ }^{30}$. However, as the results of this study show, neither the severity nor progression of the disease can be measured or interpreted using the current clinically driven staging system ${ }^{9}$. Therefore, we assert that therapy recommendations based on those criteria must be critically reviewed and interpreted.

Furthermore, it must not go unmentioned that non-surgical management in cases of infection (AAOMS stage $\mathrm{II}^{9}$ ) through the use of antibacterial treatment usually leads to a stage downshift (to AAOMS stage I) ${ }^{31,32}$, which is interpreted as a treatment success by many authors ${ }^{33}$. Such a fluent transition between those lower stages does not produce any clear information about the real osseous lesion. In addition to freedom from infection, some authors describe a decline in the mucosal lesion and sometimes even the total rehabilitation of mucosal integrity ${ }^{33-35}$. In this study, we observed a reduction in the size of the mucosal lesion between the time of study inclusion (T0) and the day of surgery (T1), but that improvement in the mucosal situation should not be misinterpreted as evidence that the disease has resolved. As can be seen from our intraoperative evaluation, the necrotic lesion was significantly larger than the mucosal lesion, with only a tenuous relationship between their sizes. In other words, the successful elimination of infection and restoration of mucosal integrity neither resolves nor revitalizes the necrotic bone.

Data from a recent longitudinal study by our working group even suggests that necrotic bone defects show a tendency to enlarge rather than diminish ${ }^{32}$. In that study, 92 patients with stage I MRONJ ${ }^{9}$ were initially treated using a standardized conservative (non-surgical) protocol of antimicrobial mouth rinsing and gel application (with chlorhexidine). Only 8 patients $(8.7 \%)$ showed complete mucosal healing and resolution of symptoms, whereas the remaining 84 (91.3\%) had persistent exposed jawbone at the end of the observation period (15.6 months). Among those 84 patients, 67 (79.8\%) showed an upshift in AAOMS stage from I to II or III, which inevitably led to operations, with extensive bone loss in 28 cases $^{32}$.

Considering that the management of stage III patients is 
known to be a major challenge and that outcomes are often worse than in earlier stages ${ }^{36}$, the AAOMS treatment recommendations are even more questionable. In a large retrospective cohort using data from more than 10 years, Ruggiero and $\mathrm{Kohn}^{36}$ observed a significant difference in outcomes with respect to the disease stage, with stage I and II disease showing a higher likelihood of better outcomes than stage III. In addition, they showed that a positive result in stage III was 28 times more likely to be achieved by surgical treatment than by non-surgical treatment ${ }^{36}$. Those results stand in total contrast to the recommendations published a year before by that same author'.

A closer look at our data shows that the necrotic lesion is 2 to 3 times larger than the mucosal lesion. The extent of the necrotic lesion in patients with only probeable bone was also comparable to that in patients with visible bone. Surgically induced resection defects of up to $53 \mathrm{~mm}$ in this trial argue in favor of early surgical treatment and against postponing surgical therapy until stage III $^{9}$ has been reached. Given that MRONJ patients are usually already seriously ill and in need of rapid continuation of their antiresorptive or oncological therapy, which is delayed by conservative, non-surgical therapy $^{37,38}$, several working groups and international guidelines argue in favor of early surgical treatment.

To summarize our findings, the clinically driven AAOMS staging system fails to reflect the actual extent of MRONJ. As a logical consequence, it is not possible to derive correct treatment recommendations from it $^{11}$. Knowledge about the extent of necrosis is indispensable to surgical treatment planning for MRONJ. Consequently, some authors recommend the use of different imaging modalities to accurately assess the extent of MRONJ preoperatively ${ }^{12}$.

Unfortunately, no consensus has been reached on the efficacy of different radiological imaging modalities in assessing the extent of a necrotic osseous lesion ${ }^{39}$. The latest metaanalysis to examine the prevalence of radiographic findings on jaws exposed to antiresorptive therapy, including jaws with MRONJ, identified only 29 studies with a total of 1,133 patients. The most frequent radiological findings were mixed lytic-sclerotic areas (73.88\%), osteolytic changes $(66.18 \%)$, osteosclerosis (65.75\%), cortical bone erosion (50.83\%), and persisting alveolar socket $(45.77 \%)^{39}$. Those authors emphasized that the overall level of evidence in the studies identified was very low. They also pointed out that no imaging modality currently allows clear differentiation between necrotic and vital bone ${ }^{39}$. That is why we assessed the extent of MRONJ in this study using an intraoperative measurement technique.

Certainly, auto-fluorescence-guided resection of necrotic bone has some drawbacks. For instance, bleeding in the operating area makes the fluorescence properties difficult to obtain, which might distort the measurements. It is also difficult to use this technique in a sterile operating theater because no currently available devices allow thermic sterilization ${ }^{22}$. However, this technique can assess the extent of necrotic bone with great clinical accuracy ${ }^{21,22}$.

Nonetheless, further efforts must be made to find radiographic modalities that can clearly differentiate between necrotic and vital bone. Such modalities could enable early diagnosis, significantly affecting the management of the underlying disease and enabling better treatment and the prevention of new potential $\operatorname{cases}^{39,40}$. By assessing the real extent of the lesion and detecting asymptomatic necrotic areas, high-resolution imaging could be helpful in individualized treatment planning ${ }^{41}$. Such imaging is necessary to define a new classification for MRONJ.

The main limitation of this study is the relatively small number of patients involved, which inevitably limits its statistical power to detect significant associations. Furthermore, only patients with stage I MRONJ ${ }^{9}$ were included in the trial. Therefore, these results can only be generalized to other patients to a limited degree. In assessing the extent of mucosal or necrotic lesions or resection defects, the measurement methods allowed only two-dimensional measurements. Because MRONJ is truly a three-dimensional problem, future studies should find a way to make three-dimensional measurements. The heterogeneous intake of antiresorptive drugs, as well anti-angiogenetic or immunomodulatory medications, might also be a limitation because our sample size was too small to allow subgroup analyses that could highlight potential differences caused by the type and administration of medications. Indeed, subgroup analyses in multifactorial regression models might be the aim of future prospective clinical trials. This research has brought to light many questions that need further investigation. Future studies should use a prospective approach to analyze a larger group of patients with all stages of MRONJ.

\section{Conclusion}

In this retrospective study, we have shown that preoperative mucosal defects (visible or probeable) in MRONJ patients do not allow exact conclusions to be drawn about the intraoperative extent of bone necrosis, and thus they provide 
inadequate information about disease severity. In comparing the visible bone and probeable bone groups, the extent of the osseous necrotic lesion in the anteroposterior direction was significantly larger in patients with a visible mucosal defect. In the transversal direction, no significant differences between those groups were found. Therefore, therapy recommendations that are partly based on the size of the mucosal defect must be critically reviewed. Therapy decisions should be made on a patient-specific basis, and they should combine clinical examination and radiological findings, always taking into account the general condition of the patient and consultation with the treating oncologist or osteologist.

\section{ORCID}

Oliver Ristow, https://orcid.org/0000-0002-3911-262X

Lena Hürtgen, https://orcid.org/0000-0002-0965-3660

Julius Moratin, https://orcid.org/0000-0002-0278-7228

Maximilian Smielowski, https://orcid.org/0000-0002-42933541

Christian Freudlsperger, https://orcid.org/0000-0002-42890457

Michael Engel, https://orcid.org/0000-0001-8573-0464

Jürgen Hoffmann, https://orcid.org/0000-0002-3286-2145

Thomas Rückschloß, https://orcid.org/0000-0002-7955-9194

\section{Authors' Contributions}

All authors contributed to the study conception, data acquisition, and design. Material preparation, data collection, and analysis were performed by T.R., O.R., and L.H. The first draft of the manuscript was written by O.R., T.R., and L.H., and all authors commented on previous versions of the manuscript. Conception and design of study and preparation of ethical approval were performed by J.M., M.S., C.F., M.E., and J.H. Patient aftercare was performed by M.S., M.E., and J.H. All authors read and approved the final manuscript.

\section{Acknowledgements}

We thank Mr. Jonathan Griffiths for language editing of this manuscript. This study was supported and funded by the Physician Scientists Program of the Medical Faculty of Heidelberg University.

\section{Ethics Approval and Consent to Participate}

All applicable international, national, and institutional guidelines for human studies were followed. This study was approved by the Ethics Committee of the University of Heidelberg (No. S-401/2020). For this type of study, formal consent was not required.

\section{Consent for Publishing Photographs}

Written informed consent was obtained from the patients for publication of this article and accompanying images.

\section{Conflict of Interest}

Thomas Rückschloß declares that he has no conflicts of interest, though he was supported by the Physician Scientists Program of the Medical Faculty of Heidelberg University. The other authors have no potential conflict of interest to disclose.

\section{References}

1. Marx RE. Pamidronate (Aredia) and zoledronate (Zometa) induced avascular necrosis of the jaws: a growing epidemic. J Oral Maxillofac Surg 2003;61:1115-7. https://doi.org/10.1016/s02782391(03)00720-1

2. Viviano M, Addamo A, Cocca S. A case of bisphosphonate-related osteonecrosis of the jaw with a particularly unfavourable course: a case report. J Korean Assoc Oral Maxillofac Surg 2017;43:272-5. https://doi.org/10.5125/jkaoms.2017.43.4.272

3. Khan AA, Morrison A, Kendler DL, Rizzoli R, Hanley DA, Felsenberg D, et al.; International Task Force on Osteonecrosis of the Jaw. Case-based review of osteonecrosis of the jaw (ONJ) and application of the international recommendations for management from the international task force on ONJ. J Clin Densitom 2017;20:8-24. https://doi.org/10.1016/j.jocd.2016.09.005

4. Kim KM, Rhee Y, Kwon YD, Kwon TG, Lee JK, Kim DY. Medication related osteonecrosis of the jaw: 2015 position statement of the Korean Society for Bone and Mineral Research and the Korean Association of Oral and Maxillofacial Surgeons. J Bone Metab 2015;22:151-65. https://doi.org/10.11005/jbm.2015.22.4.151

5. Schiegnitz E, Al-Nawas B, Hoefert S, Otto S, Pautke C, Ristow O, et al. S3-leitlinie 007/091: antiresorptiva-assoziierte kiefernekrosen (AR-ONJ). Berlin: Arbeitsgemeinschaft der Wissenschaftlichen Medizinischen Fachgesellschaften (AWMF); 2017.

6. Yarom N, Shapiro CL, Peterson DE, Van Poznak CH, Bohlke K, Ruggiero SL, et al. Medication-related osteonecrosis of the jaw: MASCC/ISOO/ASCO clinical practice guideline. J Clin Oncol 2019;37:2270-90. https://doi.org/10.1200/JCO.19.01186

7. Advisory Task Force on Bisphosphonate-Related Ostenonecrosis of the Jaws, American Association of Oral and Maxillofacial Surgeons. American Association of Oral and Maxillofacial Surgeons position paper on bisphosphonate-related osteonecrosis of the jaws. J Oral Maxillofac Surg 2007;65:369-76. https://doi.org/10.1016/ j.joms.2006.11.003 
8. Ruggiero SL, Dodson TB, Assael LA, Landesberg R, Marx RE, Mehrotra B; American Association of Oral and Maxillofacial Surgeons. American Association of Oral and Maxillofacial Surgeons position paper on bisphosphonate-related osteonecrosis of the jaws--2009 update. J Oral Maxillofac Surg 2009;67(5 Suppl):2-12. https://doi.org/10.1016/j.joms.2009.01.009

9. Ruggiero SL, Dodson TB, Fantasia J, Goodday R, Aghaloo T, Mehrotra B, et al.; American Association of Oral and Maxillofacial Surgeons. American Association of Oral and Maxillofacial Surgeons position paper on medication-related osteonecrosis of the jaw--2014 update. J Oral Maxillofac Surg 2014;72:1938-56. https://doi.org/10.1016/j.joms.2014.04.031

10. Fedele S, Bedogni G, Scoletta M, Favia G, Colella G, Agrillo A, et al. Up to a quarter of patients with osteonecrosis of the jaw associated with antiresorptive agents remain undiagnosed. $\mathrm{Br}$ J Oral Maxillofac Surg 2015;53:13-7. https://doi.org/10.1016/ j.bjoms.2014.09.001

11. Schiodt M, Otto S, Fedele S, Bedogni A, Nicolatou-Galitis O, Guggenberger R, et al. Workshop of European task force on medication-related osteonecrosis of the jaw-current challenges. Oral Dis 2019;25:1815-21. https://doi.org/10.1111/odi.13160

12. Ascani G, Campisi G, Junquera Gutierrez LM. Current controversies in classification, management, and prevention of bisphosphonate-related osteonecrosis of the jaw. Int J Dent 2014;2014:565743. https://doi.org/10.1155/2014/565743

13. Bedogni A, Fusco V, Agrillo A, Campisi G. Learning from experience. Proposal of a refined definition and staging system for bisphosphonate-related osteonecrosis of the jaw (BRONJ). Oral Dis 2012;18:621-3. https://doi.org/10.1111/j.1601-0825.2012.01903.x

14. Franco S, Miccoli S, Limongelli L, Tempesta A, Favia G, Maiorano E, et al. New dimensional staging of bisphosphonate-related osteonecrosis of the jaw allowing a guided surgical treatment protocol: long-term follow-up of 266 lesions in neoplastic and osteoporotic patients from the University of Bari. Int J Dent 2014;2014:935657. https://doi.org/10.1155/2014/935657

15. Ogura I, Kobayashi E, Nakahara K, Igarashi K, Haga-Tsujimura M, Toshima H. Quantitative SPECT/CT imaging for medicationrelated osteonecrosis of the jaw: a preliminary study using volumebased parameters, comparison with chronic osteomyelitis. Ann Nucl Med 2019;33:776-82. https://doi.org/10.1007/s12149-01901390-5

16. Okui T, Kobayashi Y, Tsujimoto M, Satoh K, Toyama H, Matsuo K. Quantitative evaluation of anti-resorptive agent-related osteonecrosis of the jaw using bone single photon emission computed tomography in clinical settings: relationship between clinical stage and imaging. Ann Nucl Med 2020;34:620-8. https://doi.org/10.1007/ s12149-020-01485-4

17. Bisdas S, Chambron Pinho N, Smolarz A, Sader R, Vogl TJ, Mack MG. Biphosphonate-induced osteonecrosis of the jaws: CT and MRI spectrum of findings in 32 patients. Clin Radiol 2008;63:717. https://doi.org/10.1016/j.crad.2007.04.023

18. Devlin H, Greenwall-Cohen J, Benton J, Goodwin TL, Littlewood A, Horner K. Detecting the earliest radiological signs of bisphosphonate-related osteonecrosis. Br Dent J 2018;224:26-31. https:// doi.org/10.1038/sj.bdj.2017.1001

19. Otto S, Pautke C, Van den Wyngaert T, Niepel D, Schiødt M. Medication-related osteonecrosis of the jaw: prevention, diagnosis and management in patients with cancer and bone metastases. Cancer Treat Rev 2018;69:177-87. https://doi.org/10.1016/ j.ctrv.2018.06.007

20. Assaf AT, Zrnc TA, Riecke B, Wikner J, Zustin J, Friedrich RE, et al. Intraoperative efficiency of fluorescence imaging by Visually Enhanced Lesion Scope (VELscope) in patients with bisphosphonate related osteonecrosis of the jaw (BRONJ). J Craniomaxillofac Surg 2014;42:e157-64. https://doi.org/10.1016/j.jcms.2013.07.014

21. Ristow O, Otto S, Geiß C, Kehl V, Berger M, Troeltzsch M, et al. Comparison of auto-fluorescence and tetracycline fluorescence for guided bone surgery of medication-related osteonecrosis of the jaw: a randomized controlled feasibility study. Int J Oral Maxillofac Surg 2017;46:157-66. https://doi.org/10.1016/j.ijom.2016.10.008

22. Ristow O, Nehrbass D, Zeiter S, Arens D, Moratin J, Pautke C, et al. Differences between auto-fluorescence and tetracyclinefluorescence in medication-related osteonecrosis of the jaw-a preclinical proof of concept study in the mini-pig. Clin Oral Investig 2020;24:4625-37. https://doi.org/10.1007/s00784-020-03332-2

23. Ristow O, Rückschloß T, Bodem J, Berger M, Bodem E, Kargus $\mathrm{S}$, et al. Double-layer closure techniques after bone surgery of medication-related osteonecrosis of the jaw - a single center cohort study. J Craniomaxillofac Surg 2018;46:815-24. https://doi. org/10.1016/j.jcms.2018.03.005

24. Fedele S, Porter SR, D'Aiuto F, Aljohani S, Vescovi P, Manfredi $\mathrm{M}$, et al. Nonexposed variant of bisphosphonate-associated osteonecrosis of the jaw: a case series. Am J Med 2010;123:1060-4. https://doi.org/10.1016/j.amjmed.2010.04.033

25. Schiodt M, Reibel J, Oturai P, Kofod T. Comparison of nonexposed and exposed bisphosphonate-induced osteonecrosis of the jaws: a retrospective analysis from the Copenhagen cohort and a proposal for an updated classification system. Oral Surg Oral Med Oral Pathol Oral Radiol 2014;117:204-13. https://doi.org/10.1016/ j.oooo.2013.10.010

26. Nicolatou-Galitis O, Papadopoulou E, Vardas E, Kouri M, Galiti D, Galitis E, et al. Alveolar bone histological necrosis observed prior to extractions in patients, who received bone-targeting agents. Oral Dis 2020;26:955-66. https://doi.org/10.1111/odi.13294

27. Ristow O, Rückschloß T, Moratin J, Müller M, Kühle R, Dominik $\mathrm{H}$, et al. Wound closure and alveoplasty after preventive tooth extractions in patients with antiresorptive intake-a randomized pilot trial. Oral Dis 2021;27:532-46. https://doi.org/10.1111/odi.13556

28. Yarom N, Fedele S, Lazarovici TS, Elad S. Is exposure of the jawbone mandatory for establishing the diagnosis of bisphosphonate-related osteonecrosis of the jaw? J Oral Maxillofac Surg 2010;68:705. https://doi.org/10.1016/j.joms.2009.07.086

29. Assaf AT, Zrnc TA, Remus CC, Adam G, Zustin J, Heiland M, et al. Intraindividual comparison of preoperative (99m)Tc-MDP $\mathrm{SPECT} / \mathrm{CT}$ and intraoperative and histopathological findings in patients with bisphosphonate- or denosumab-related osteonecrosis of the jaw. J Craniomaxillofac Surg 2015;43:1461-9. https://doi. org/10.1016/j.jcms.2015.06.025

30. Khan AA, Morrison A, Hanley DA, Felsenberg D, McCauley LK, O'Ryan F, et al.; International Task Force on Osteonecrosis of the Jaw. Diagnosis and management of osteonecrosis of the jaw: a systematic review and international consensus. J Bone Miner Res 2015;30:3-23. https://doi.org/10.1002/jbmr.2405

31. Hayashida S, Soutome S, Yanamoto S, Fujita S, Hasegawa $\mathrm{T}$, Komori T, et al. Evaluation of the treatment strategies for medication-related osteonecrosis of the jaws (MRONJ) and the factors affecting treatment outcome: a multicenter retrospective study with propensity score matching analysis. J Bone Miner Res 2017;32:2022-9. https://doi.org/10.1002/jbmr.3191

32. Ristow O, Rückschloß T, Müller M, Berger M, Kargus S, Pautke $\mathrm{C}$, et al. Is the conservative non-surgical management of medication-related osteonecrosis of the jaw an appropriate treatment option for early stages? A long-term single-center cohort study. J Craniomaxillofac Surg 2019;47:491-9. https://doi.org/10.1016/ j.jcms.2018.12.014

33. Bodem JP, Kargus S, Engel M, Hoffmann J, Freudlsperger C. Value of nonsurgical therapeutic management of stage I bisphosphonate-related osteonecrosis of the jaw. J Craniomaxillofac Surg 2015;43:1139-43. https://doi.org/10.1016/j.jcms.2015.05.019

34. Albanese M, Zotti F, Capocasale G, Bonetti S, Lonardi F, Nocini $\mathrm{PF}$. Conservative non-surgical management in medication related osteonecrosis of the jaw: a retrospective study. Clin Exp Dent Res 2020;6:512-8. https://doi.org/10.1002/cre2.303

35. Moretti F, Pelliccioni GA, Montebugnoli L, Marchetti C. A pro- 
spective clinical trial for assessing the efficacy of a minimally invasive protocol in patients with bisphosphonate-associated osteonecrosis of the jaws. Oral Surg Oral Med Oral Pathol Oral Radiol Endod 2011;112:777-82. https://doi.org/10.1016/ j.tripleo.2011.07.004

36. Ruggiero SL, Kohn N. Disease stage and mode of therapy are important determinants of treatment outcomes for medicationrelated osteonecrosis of the jaw. J Oral Maxillofac Surg 2015;73(12 Suppl):S94-100. https://doi.org/10.1016/j.joms.2015.09.024

37. Kang SH, Park SJ, Kim MK. The effect of bisphosphonate discontinuation on the incidence of postoperative medication-related osteonecrosis of the jaw after tooth extraction. J Korean Assoc Oral Maxillofac Surg 2020;46:78-83. https://doi.org/10.5125/jkaoms.2020.46.1.78

38. Kim YH, Lee HK, Song SI, Lee JK. Drug holiday as a prognostic factor of medication-related osteonecrosis of the jaw. J Korean Assoc Oral Maxillofac Surg 2014;40:206-10. https://doi.org/10.5125/ jkaoms.2014.40.5.206

39. Dutra KL, Haas LF, Zimmermann GS, Melo G, Minamisako MC, Flores-Mir C, et al. Prevalence of radiographic findings on jaws exposed to antiresorptive therapy: a meta-analysis. Dentomaxillofac
Radiol 2019;48:20180112. https://doi.org/10.1259/dmfr.20180112

40. Moreno-Rabié C, Gaêta-Araujo H, Oliveira-Santos C, Politis C, Jacobs R. Early imaging signs of the use of antiresorptive medication and MRONJ: a systematic review. Clin Oral Investig 2020;24:2973-89. https://doi.org/10.1007/s00784-020-03423-0

41. Tsuchimochi M, Kurabayashi T. Symposium: imaging modalities for drug-related osteonecrosis of the jaw (1), role of imaging in drug-related osteonecrosis of the jaw: an up-to-date review (secondary publication). Jpn Dent Sci Rev 2019;55:1-4. https://doi. org/10.1016/j.jdsr.2018.11.004

How to cite this article: Ristow O, Hürtgen L, Moratin J, Smielowski M, Freudlsperger C, Engel M, et al. A critical assessment of the medication-related osteonecrosis of the jaw classification in stage I patients: a retrospective analysis. J Korean Assoc Oral Maxillofac Surg 2021;47:99-111. https://doi.org/10.5125/jkaoms.2021.47.2.99 